\title{
"QUASI"-NORM OF AN ARITHMETICAL CONVOLUTION OPERATOR AND THE ORDER OF THE RIEMANN ZETA FUNCTION
}

\author{
Titus Hilberdink
}

\begin{abstract}
In this paper we study Dirichlet convolution with a given arithmetical function $f$ as a linear mapping $\varphi_{f}$ that sends a sequence $\left(a_{n}\right)$ to $\left(b_{n}\right)$ where $b_{n}=\sum_{d \mid n} f(d) a_{n / d}$. We investigate when this is a bounded operator on $l^{2}$ and find the operator norm. Of particular interest is the case $f(n)=n^{-\alpha}$ for its connection to the Riemann zeta function on the line $\Re s=\alpha$. For $\alpha>1, \varphi_{f}$ is bounded with $\left\|\varphi_{f}\right\|=\zeta(\alpha)$.

For the unbounded case, we show that $\varphi_{f}: \mathcal{M}^{2} \rightarrow \mathcal{M}^{2}$ where $\mathcal{M}^{2}$ is the subset of $l^{2}$ of multiplicative sequences, for many $f \in \mathcal{M}^{2}$. Consequently, we study the 'quasi'-norm

$$
\sup _{\substack{\|a\|=T \\ a \in \mathcal{M}^{2}}} \frac{\left\|\varphi_{f} a\right\|}{\|a\|}
$$

for large $T$, which measures the 'size' of $\varphi_{f}$ on $\mathcal{M}^{2}$. For the $f(n)=n^{-\alpha}$ case, we show this quasi-norm has a striking resemblance to the conjectured maximal order of $|\zeta(\alpha+i T)|$ for $\alpha>\frac{1}{2}$.
\end{abstract} Keywords: Dirichlet convolution, maximal order of the Riemann zeta function.

\section{Introduction}

Given an arithmetical function $f(n)$, the mapping $\varphi_{f}$ sends $\left(a_{n}\right)_{n \in \mathbb{N}}$ to $\left(b_{n}\right)_{n \in \mathbb{N}}$, where

$$
b_{n}=\sum_{d \mid n} f(d) a_{n / d} .
$$

Writing $a=\left(a_{n}\right), \varphi_{f}$ maps $a$ to $f * a$ where $*$ is Dirichlet convolution. This is a "matrix" mapping, where the matrix, say $M(f)$, is of "multiplicative Toeplitz" type; that is,

$$
M(f)=\left(a_{i j}\right)_{i, j \geqslant 1}
$$

where $a_{i j}=f(i / j)$ and $f$ is supported on the natural numbers (see, for example, $[6],[7])$.

2010 Mathematics Subject Classification: primary: 11N37; secondary: 11M06 
Toeplitz matrices (whose $i j^{\text {th }}$-entry is a function of $i-j$ ) are most usefully studied in terms of a "symbol" (the function whose Fourier coefficients make up the matrix). Analogously, the Multiplicative Toeplitz matrix $M(f)$ has as symbol the Dirichlet series

$$
\sum_{n=1}^{\infty} f(n) n^{i t}
$$

Our particular interest is naturally the case $f(n)=n^{-\alpha}$ when the symbol is $\zeta(\alpha-i t)$. We are especially interested how and to what extent properties of the mapping relate to properties of the symbol for $\alpha \leqslant 1$.

These type of mappings were considered by various authors (for example Wintner [15]) and most notably Toeplitz [13], [14] (although somewhat indirectly, through his investigations of so-called " $D$-forms"). In essence, Toeplitz proved that $\varphi_{f}: l^{2} \rightarrow l^{2}$ is bounded if and only if $\sum_{n=1}^{\infty} f(n) n^{-s}$ is defined and bounded for all $\Re s>0$. In particular, if $f(n) \geqslant 0$ then $\varphi_{f}$ is bounded on $l^{2}$ if and only if $f \in l^{1}$; furthermore, the operator norm is $\left\|\varphi_{f}\right\|=\|f\|_{1}$. We prove this in Theorem 1.1 following Toeplitz's original idea. For example, for $f(n)=n^{-\alpha}, \varphi_{f}$ is bounded on $l^{2}$ for $\alpha>1$ with operator norm $\zeta(\alpha)$. In this special case, the mapping was studied in [7] for $\alpha \leqslant 1$ when it is unbounded on $l^{2}$ by estimating the behaviour of the quantity

$$
\Phi_{f}(N)=\sup _{\|a\|_{2}=1}\left(\sum_{n=1}^{N}\left|b_{n}\right|^{2}\right)^{1 / 2}
$$

for large $N$. Approximate formulas for $\Phi_{f}(N)$ were obtained and it was shown that, for $\frac{1}{2}<\alpha \leqslant 1, \Phi_{f}(N)$ is a lower bound for $\max _{1 \leqslant t \leqslant T}|\zeta(\alpha+i t)|$ with $N=T^{\lambda}$ (some $\lambda>0$ depending on $\alpha$ only). In this way, it was proven that the measure of the set

$$
\left\{t \in[1, T]:|\zeta(1+i t)| \geqslant e^{\gamma} \log \log T-A\right\}
$$

is at least $T \exp \left\{-a \frac{\log T}{\log \log T}\right\}$ (some $a>0$ ) for $A$ sufficiently large, while for $\frac{1}{2}<$ $\alpha<1$ one has

$$
\max _{t \leqslant T}|\zeta(\alpha+i t)| \geqslant \exp \left\{\frac{c(\log T)^{1-\alpha}}{\log \log T}\right\}
$$

for some $c>0$ depending on $\alpha$ only, as well providing an estimate for how often $|\zeta(\alpha+i T)|$ is as large as the right-hand side above. The method is akin to Soundararajan's "resonance" method and incidentally shows the limitation of this approach for $\alpha>\frac{1}{2}$ since $|\zeta(\alpha+i T)|$ is known to be of larger order.

In this paper we study the unbounded case in a different way, by restricting the domain. Thus in section 2 , we show that for many multiplicative $f$, in particular for $f$ completely multiplicative, $\varphi_{f}\left(\mathcal{M}^{2}\right) \subset \mathcal{M}^{2}$ even though $\varphi_{f}\left(l^{2}\right) \not \subset l^{2}$. Here $\mathcal{M}^{2}$ is the set of multiplicative functions in $l^{2}$. As a result we consider, for such $f$, the "quasi"-norm

$$
M_{f}(T)=\sup _{\substack{\|a\|=T \\ a \in \mathcal{M}^{2}}} \frac{\left\|\varphi_{f} a\right\|}{\|a\|}
$$


and obtain approximate formulae for large $T$ (here $\|\cdot\|$ is the usual $l^{2}$-norm). We find that for the particular case $f(n)=n^{-\alpha}\left(\alpha>\frac{1}{2}\right)$, this quasi-norm has a striking similarity to the conjectured maximal order of $|\zeta(\alpha+i T)|$. For example, with $\alpha=1$ (i.e. $f(n)=1 / n$ ) we prove

$$
M_{f}(T)=e^{\gamma}(\log \log T+\log \log \log T+2 \log 2-1)+o(1)
$$

while for $\frac{1}{2}<\alpha<1$

$$
\log M_{f}(T) \sim \frac{B\left(\frac{1}{\alpha}, 1-\frac{1}{2 \alpha}\right)^{\alpha}}{(1-\alpha) 2^{\alpha}} \frac{(\log T)^{1-\alpha}}{(\log \log T)^{\alpha}},
$$

where $B(x, y)$ is the Beta function. Writing $Z_{\alpha}(T)=\max _{1 \leqslant t \leqslant T}|\zeta(\alpha+i t)|$, Granville and Soundararajan [3] proved that $Z_{1}(T)$ is at least as large as $(0.2)$ minus a $\log \log \log \log T$ term for some arbitrarily large $T$ and they conjectured that it equals (0.2) (possibly with a different constant term). For $\frac{1}{2}<\alpha<1$, Montgomery [9] found

$$
\log Z_{\alpha}(T) \geqslant \frac{\sqrt{\alpha-1 / 2}}{20} \frac{(\log T)^{1-\alpha}}{(\log \log T)^{\alpha}}
$$

and, using a heuristic argument, conjectured that this is (apart from the constant) the correct order of $\log Z_{\alpha}(T)$. Further, in a recent paper (see [8]), Lamzouri suggests $\log Z_{\alpha}(T) \sim C(\alpha)(\log T)^{1-\alpha}(\log \log T)^{-\alpha}$ with some specific constant $C(\alpha)$ (see also the remark after Theorem 3.1).

Similarly one can study the quantity

$$
m_{f}(T)=\inf _{\substack{\|a\|=T \\ a \in \mathcal{M}^{2}}} \frac{\left\|\varphi_{f} a\right\|}{\|a\|} .
$$

With $f(n)=n^{-\alpha}$ this is shown to behave like the known and conjectured minimal order of $|\zeta(\alpha+i T)|$ for $\alpha>\frac{1}{2}$. It should be stressed here that, unlike the case of $\Phi_{f}(N)$ which was shown to be a lower bound for $Z_{\alpha}(T)$ in [7], we have not proved any connection between $\zeta(\alpha+i T)$ and $M_{f}(T)$. Even to show $M_{f}(T)$ is a lower bound would be very interesting.

Our results, though motivated by the special case $f(n)=n^{-\alpha}$, extend naturally to completely multiplicative $f$ for which $\left.f\right|_{\mathbb{P}}$ is regularly varying (see section 2 for the definition).

Addendum. I would like to thank the anonymous referee for some useful comments and for pointing out a recent paper by Aistleitner and Seip [1]. They deal with an optimization problem which is different yet curiously similar. The function $\exp \left\{c_{\alpha}(\log T)^{1-\alpha}(\log \log T)^{-\alpha}\right\}$ appears in the same way, although their $c_{\alpha}$ is expected to remain bounded as $\alpha \rightarrow \frac{1}{2}$. It would be interesting to investigate any links further. 


\section{Bounded operators}

Notation. Let $l^{1}$ and $l^{2}$ denote the usual spaces of sequences $\left(a_{n}\right)_{n \in \mathbb{N}}$, with norms $\|a\|_{1}=\sum\left|a_{n}\right|$ and $\|a\|_{2}=\left(\sum\left|a_{n}\right|^{2}\right)^{1 / 2}$ respectively. After section 1 we shall, for ease of notation, just write $\|\cdot\|$ for $\|\cdot\|_{2}$ since it is the norm we will use.

A linear mapping $\varphi: l^{2} \rightarrow l^{2}$ is bounded if there exists $C>0$ such that $\|\varphi x\|_{2} \leqslant C\|x\|_{2}$ for all $x \in l^{2}$. As such, we define the operator norm by

$$
\|\varphi\|=\sup _{x \neq 0} \frac{\|\varphi x\|_{2}}{\|x\|_{2}}=\sup _{\|x\|_{2}=1}\|\varphi x\|_{2} .
$$

We shall assume from now on that $f(n) \geqslant 0$ for all $n \in \mathbb{N}$. We are particularly interested in the case where $\varphi_{f}$ acts on $l^{2}$. Define the function

$$
\Phi_{f}(N)=\sup _{\|a\|_{2}=1} \sqrt{\sum_{n \leqslant N}\left|b_{n}\right|^{2}}
$$

where $b_{n}$ is given in terms of $a_{n}$ by (0.1). Note that the supremum will occur when $a_{n} \geqslant 0$ for all $n$ and when $\sum_{n \leqslant N} a_{n}^{2}=1$.

Suppose now that $f \in l^{1}$; i.e. $\|f\|_{1}=\sum_{n=1}^{\infty} f(n)<\infty$. Then

$\left|b_{n}\right|^{2}=\left|\sum_{d \mid n} \sqrt{f(d)} \cdot \sqrt{f(d)} a_{n / d}\right|^{2} \leqslant \sum_{d \mid n} f(d) \sum_{d \mid n} f(d)\left|a_{n / d}\right|^{2} \leqslant\|f\|_{1} \sum_{d \mid n} f(d)\left|a_{n / d}\right|^{2}$.

Hence

$$
\sum_{n \leqslant N}\left|b_{n}\right|^{2} \leqslant\|f\|_{1} \sum_{n \leqslant N} \sum_{d \mid n} f(d)\left|a_{n / d}\right|^{2}=\|f\|_{1} \sum_{d \leqslant N} f(d) \sum_{n \leqslant N / d}\left|a_{n}\right|^{2} \leqslant\|f\|_{1}^{2}\|a\|_{2}^{2} .
$$

Thus

$$
\Phi_{f}(N) \leqslant\|f\|_{1} .
$$

Following Toeplitz [14], we show that this inequality is sharp.

Theorem 1.1. Let $f$ be a non-negative arithmetical function and $f \in l^{1}$. Then $\Phi_{f}(N) \rightarrow\|f\|_{1}$ as $N \rightarrow \infty$. Thus $\varphi_{f}: l^{2} \rightarrow l^{2}$ is bounded if and only if $f \in l^{1}$, in which case $\left\|\varphi_{f}\right\|=\|f\|_{1}$.

Proof. After (1.1), and since $\Phi_{f}(N)$ increases with $N$, we need only provide a lower bound for an infinite sequence of $N$ s. Let $a_{n}=d(N)^{-\frac{1}{2}}$ for $n \mid N$ and zero otherwise ( $N$ to be chosen later), where $d(\cdot)$ is the divisor function. Thus $a_{1}^{2}+\ldots+a_{N}^{2}=1$ and

$$
\Phi_{f}(N) \geqslant \sum_{n \leqslant N} a_{n} b_{n}=\frac{1}{d(N)} \sum_{n \mid N} \sum_{d \mid n} f(d)=\frac{1}{d(N)} \sum_{d \mid N} f(d) d\left(\frac{N}{d}\right)
$$


say. We choose $N$ such that it has all divisors $d$ up to some (large) number, and that $\frac{d(N / d)}{d(N)}$ is close to 1 for each such divisor $d$ of $N$. Take $N$ of the form

$$
N=\prod_{p \leqslant P} p^{\alpha_{p}}, \quad \text { where } \alpha_{p}=\left[\frac{\log P}{\log p}\right] .
$$

Thus every natural number up to $P$ is a divisor of $N$. For a divisor $d=\prod_{p \leqslant P} p^{\beta_{p}}$ of $N$, we have

$$
\frac{d(N / d)}{d(N)}=\prod_{p \leqslant P}\left(1-\frac{\beta_{p}}{\alpha_{p}+1}\right) .
$$

If we take $d \leqslant \sqrt{\log P}$, then $p^{\beta_{p}} \leqslant \sqrt{\log P}$ for every prime divisor $p$ of $d$. Hence, for such $p, \beta_{p} \leqslant \frac{\log \log P}{2 \log p}$ and $\beta_{p}=0$ if $p>\sqrt{\log P}$. Thus for $d \leqslant \sqrt{\log P}$,

$$
\begin{aligned}
\frac{d(N / d)}{d(N)} & =\prod_{p \leqslant \sqrt{\log P}}\left(1-\frac{\beta_{p}}{\alpha_{p}+1}\right) \\
& \geqslant \prod_{p \leqslant \sqrt{\log P}}\left(1-\frac{\log \log P}{2 \log P}\right)=\left(1-\frac{\log \log P}{2 \log P}\right)^{\pi(\sqrt{\log P})},
\end{aligned}
$$

where $\pi(x)$ is the number of primes up to $x$. Since $\pi(x)=O\left(\frac{x}{\log x}\right)$, it follows that for all $P$ sufficiently large, the expression in (1.2) is at least

$$
\left(1-\frac{A}{\sqrt{\log P}}\right) \sum_{d \leqslant \sqrt{\log P}} f(d)
$$

for some constant $A$. The sum can be made as close to $\|f\|_{1}$ as we please by increasing $P$.

\section{Unbounded operators on $l^{2}$}

Now we investigate when $\varphi_{f}$ is unbounded on $l^{2}$ (i.e. $f \notin l^{1}$ ). In a similar generalisation of Theorem 1.1 of [7], one can readily show that both $\varphi_{f}: l^{1} \rightarrow l^{2}$ and $\varphi_{f}: l^{2} \rightarrow l^{\infty}$ are bounded if and only if $f \in l^{2}$, with $\left\|\varphi_{f}\right\|=\|f\|_{2}$ in either case. So here we shall assume that $f \in l^{2} \backslash l^{1}$. In the appendix we see that, for all cases of interest at least, if $f \notin l^{2}$, then $\varphi_{f} a \notin l^{2}$ for all $a$ except $a=0$.

For unbounded operators, there are different ways of measuring the "unboundedness". One way, which was done in [7] for the case $f(n)=n^{-\alpha}$, is to restrict the range by looking at a restricted norm; i.e. by considering $\Phi_{f}(N)$ for given $N$. Another way is to restrict the domain to a set $S$ say, such that $\varphi_{f}(S) \subset l^{2}$ and to consider the size of

$$
\sup _{\substack{a \in S \\\|a\|=N}} \frac{\left\|\varphi_{f} a\right\|}{\|a\|} \quad \text { for large } N .
$$

For $f$ completely multiplicative one is naturally led to consider $S=\mathcal{M}^{2}$ - the set of square summable multiplicative functions. It is also natural to consider regularly varying functions. 
Regular Variation. A function $\ell:[A, \infty) \rightarrow \mathbb{R}$ is regularly varying of index $\rho$ if it is measurable and

$$
\ell(\lambda x) \sim \lambda^{\rho} \ell(x) \quad \text { as } x \rightarrow \infty \text { for every } \lambda>0
$$

(see [2] for a detailed treatise on the subject). For example, $x^{\rho}(\log x)^{\tau}$ is regularlyvarying of index $\rho$ for any $\tau$. The Uniform Convergence Theorem says that the above asymptotic formula is automatically uniform for $\lambda$ in compact subsets of $(0, \infty)$. Note that every regularly varying function of non-zero index is asymptotic to one which is strictly monotonic and continuous. We shall make use of Karamata's Theorem: for $\ell$ regularly varying of index $\rho$,

$$
\int^{x} \ell \sim \frac{x \ell(x)}{\rho+1} \quad \text { if } \rho>-1, \quad \int_{x}^{\infty} \ell \sim-\frac{x \ell(x)}{\rho+1} \quad \text { if } \rho<-1,
$$

while if $\rho=-1, \int^{x} \ell$ is slowly varying (regularly varying with index 0 ) and $\int^{x} \ell \succ$ $x \ell(x)$.

Notation. Let $\mathcal{M}^{2}$ and $\mathcal{M}_{c}^{2}$ denote the subsets of $l^{2}$ of multiplicative and completely multiplicative functions respectively. Further, write $\mathcal{M}^{2}+$ for the nonnegative members of $\mathcal{M}^{2}$ and similarly for $\mathcal{M}_{c}^{2}+$.

\subsection{The size of $\left\|\varphi_{f}\right\|$ on $\mathcal{M}^{2}$}

Now we consider $\varphi_{f}$ on the subset $\mathcal{M}^{2}$ of multiplicative functions in $l^{2}$. We suppose, as in section 2, that $f \in l^{2} \backslash l^{1}$ so that $\varphi_{f}$ is unbounded. This implies there exist $a \in l^{2}$ such that $\varphi_{f}(a) \notin l^{2}$ (by the closed graph theorem). However, if $f$ is multiplicative then, as we shall see, $\varphi_{f}\left(\mathcal{M}^{2}\right) \subset l^{2}$ in many cases (and hence $\left.\varphi_{f}\left(\mathcal{M}^{2}\right) \subset \mathcal{M}^{2}\right)$.

Lemma 2.1. Let $f, g \in \mathcal{M}^{2}$ be non-negative. Then $f * g \in \mathcal{M}^{2}$ if and only if

$$
\sum_{p} \sum_{m, n \geqslant 1} \sum_{k=0}^{\infty} f\left(p^{m}\right) g\left(p^{n}\right) f\left(p^{m+k}\right) g\left(p^{n+k}\right) \quad \text { converges. }
$$

Proof. Let $h=f * g$. Since $h$ is multiplicative,

$$
\sum_{n=1}^{\infty} h(n)^{2}<\infty \Longleftrightarrow \sum_{p} \sum_{k \geqslant 1} h\left(p^{k}\right)^{2}<\infty
$$

Let $k \geqslant 1$ and $p$ prime. Then

$$
h\left(p^{k}\right)=\sum_{r=0}^{k} f\left(p^{r}\right) g\left(p^{k-r}\right)=f\left(p^{k}\right)+g\left(p^{k}\right)+\sum_{r=1}^{k-1} f\left(p^{r}\right) g\left(p^{k-r}\right) .
$$

Using the inequality $a^{2}+b^{2}+c^{2} \leqslant(a+b+c)^{2} \leqslant 3\left(a^{2}+b^{2}+c^{2}\right)$ we have

$$
\left(\sum_{r=1}^{k-1} f\left(p^{r}\right) g\left(p^{k-r}\right)\right)^{2} \leqslant h\left(p^{k}\right)^{2} \leqslant 3 f\left(p^{k}\right)^{2}+3 g\left(p^{k}\right)^{2}+3\left(\sum_{r=1}^{k-1} f\left(p^{r}\right) g\left(p^{k-r}\right)\right)^{2} .
$$


Since $\sum_{p, k \geqslant 1} f\left(p^{k}\right)^{2}$ and $\sum_{p, k \geqslant 1} g\left(p^{k}\right)^{2}$ converge we find that $\sum_{p, k \geqslant 1} h\left(p^{k}\right)^{2}$ converges if and only if

$$
\sum_{p} \sum_{k=2}^{\infty}\left(\sum_{r=1}^{k-1} f\left(p^{r}\right) g\left(p^{k-r}\right)\right)^{2} \quad \text { converges. }
$$

But

$$
\begin{aligned}
\sum_{k=2}^{\infty}\left(\sum_{r=1}^{k-1} f\left(p^{r}\right) g\left(p^{k-r}\right)\right)^{2} & =\sum_{k=1}^{\infty} \sum_{1 \leqslant r, s \leqslant k} f\left(p^{r}\right) f\left(p^{s}\right) g\left(p^{k-r+1}\right) g\left(p^{k-s+1}\right) \\
& \leqslant 2 \sum_{k=1}^{\infty} \sum_{s=1}^{k} \sum_{r=1}^{s} f\left(p^{r}\right) f\left(p^{s}\right) g\left(p^{k-r+1}\right) g\left(p^{k-s+1}\right) \\
& =2 \sum_{r=1}^{\infty} \sum_{k=1}^{\infty} \sum_{s=0}^{\infty} f\left(p^{r}\right) f\left(p^{s+r}\right) g\left(p^{k+s}\right) g\left(p^{k}\right) .
\end{aligned}
$$

On the other hand, the RHS of (2.2) is greater than

$$
\sum_{k=1}^{\infty} \sum_{s=1}^{k} \sum_{r=1}^{s} f\left(p^{r}\right) f\left(p^{s}\right) g\left(p^{k-r+1}\right) g\left(p^{k-s+1}\right) .
$$

Hence $h \in \mathcal{M}^{2}$ if and only if

$$
\sum_{p} \sum_{m, n \geqslant 1} \sum_{k=0}^{\infty} f\left(p^{m}\right) g\left(p^{n}\right) f\left(p^{m+k}\right) g\left(p^{n+k}\right) \quad \text { converges. }
$$

Let $\mathcal{M}_{0}^{2}$ denote the set of $\mathcal{M}^{2}$ functions $f$ for which $f * g \in \mathcal{M}^{2}$ whenever $g \in \mathcal{M}^{2}$; that is,

$$
\mathcal{M}_{0}^{2}=\left\{f \in \mathcal{M}^{2}: g \in \mathcal{M}^{2} \Longrightarrow f * g \in \mathcal{M}^{2}\right\} .
$$

Thus for $f \in \mathcal{M}_{0}^{2}, \varphi_{f}\left(\mathcal{M}^{2}\right) \subset \mathcal{M}^{2}$. We shall see that it may happen that $f, g \in \mathcal{M}^{2}$ but $f * g \notin \mathcal{M}^{2}$. So $\mathcal{M}_{0}^{2} \neq \mathcal{M}^{2}$. The following gives a criterion for multiplicative functions to be in $\mathcal{M}_{0}^{2}$.

Proposition 2.1. Let $f \in \mathcal{M}^{2}$ be such that $\sum_{k=1}^{\infty}\left|f\left(p^{k}\right)\right|$ converges for every prime $p$ and that $\sum_{k=1}^{\infty}\left|f\left(p^{k}\right)\right| \leqslant A$ for some constant $A$ independent of $p$. Then $f \in \mathcal{M}_{0}^{2}$.

On the other hand, if $f \in \mathcal{M}^{2}$ with $f \geqslant 0$ and for some prime $p_{0}, f\left(p_{0}^{k}\right)$ decreases with $k$ and $\sum_{k=1}^{\infty} f\left(p_{0}^{k}\right)$ diverges, then $f \notin \mathcal{M}_{0}^{2}$.

Proof. Without loss of generality we can take $f \geqslant 0$. Let $g \in \mathcal{M}^{2}$ (again w.l.o.g. $g \geqslant 0)$ with $\alpha_{p}=\sum_{k=1}^{\infty} g\left(p^{k}\right)^{2}$. Thus $\sum_{p} \alpha_{p}$ converges. By the Cauchy-Schwarz inequality,

$$
\left(\sum_{n=1}^{\infty} g\left(p^{n}\right) g\left(p^{n+k}\right)\right)^{2} \leqslant \sum_{n=1}^{\infty} g\left(p^{n}\right)^{2} \sum_{n=1}^{\infty} g\left(p^{n+k}\right)^{2} \leqslant \alpha_{p} \alpha_{p}=\alpha_{p}^{2}
$$


Thus by Lemma $2.1, f * g \in \mathcal{M}^{2}$ if

$$
\sum_{p} \alpha_{p} \sum_{m=1}^{\infty} f\left(p^{m}\right) \sum_{k=0}^{\infty} f\left(p^{m+k}\right) \quad \text { converges. }
$$

By assumption, the inner sum over $k$ is bounded by a constant (independent of $p$ ), and hence so is the sum over $m$. This implies the convergence of the above. Hence $f * g \in \mathcal{M}^{2}$.

Now suppose $\sum_{k=1}^{\infty} f\left(p_{0}^{k}\right)$ diverges for some prime $p_{0}$. Then with $g \in \mathcal{M}^{2}$ and $g\left(p_{0}^{k}\right)$ decreasing (to zero) we have

$$
(f * g)\left(p_{0}^{k}\right)=\sum_{r=0}^{k} f\left(p_{0}^{r}\right) g\left(p_{0}^{k-r}\right) \geqslant g\left(p_{0}^{k}\right) \sum_{r=0}^{k} f\left(p_{0}^{r}\right)=g\left(p_{0}^{k}\right) c_{k},
$$

where $c_{k} \nearrow \infty$. Thus $\sum_{k}(f * g)\left(p_{0}^{k}\right)^{2} \geqslant \sum_{k} g\left(p_{0}^{k}\right)^{2} c_{k}^{2}$. But we can always choose $g\left(p_{0}^{k}\right)$ decreasing so that $\sum_{k} g\left(p_{0}^{k}\right)^{2}$ converges while, for the given sequence $c_{k}$, $\sum_{k} g\left(p_{0}^{k}\right)^{2} c_{k}^{2}$ diverges. (Choose $g\left(p_{0}^{k}\right)^{2}=\frac{1}{c_{k-1}}-\frac{1}{c_{k}}$.)

Thus $f * g \notin \mathcal{M}^{2}$; i.e. $f \notin \mathcal{M}_{0}^{2}$.

Thus, in particular, $\mathcal{M}_{c}^{2} \subset \mathcal{M}_{0}^{2}$. For $f \in \mathcal{M}_{c}^{2}$ if and only if $|f(p)|<1$ for all primes $p$ and $\sum_{p}|f(p)|^{2}<\infty$. Thus

$$
\sum_{k=1}^{\infty}\left|f\left(p^{k}\right)\right|=\frac{|f(p)|}{1-|f(p)|} \leqslant A,
$$

independent of $p$ (since $f(p) \rightarrow 0$ ).

The "quasi-norm" $M_{\boldsymbol{f}}(\boldsymbol{T})$. Let $f \in \mathcal{M}_{0}^{2}$. From above we see that $\varphi_{f}\left(\mathcal{M}^{2}\right) \subset$ $\mathcal{M}^{2}$ but, typically, $\varphi_{f}$ is not "bounded" on $\mathcal{M}^{2}$ (if $f \notin l^{1}$ ) in the sense that $\left\|\varphi_{f} a\right\| /\|a\|$ is not bounded by a constant for all $a \in \mathcal{M}^{2}$. It therefore makes sense to define, for $T \geqslant 1$,

$$
M_{f}(T)=\sup _{\substack{a \in \mathcal{M}^{2} \\\|a\|=T}} \frac{\left\|\varphi_{f} a\right\|}{\|a\|} .
$$

We aim to find the behaviour of $M_{f}(T)$ for large $T$.

We shall consider $f$ completely multiplicative and such that $\left.f\right|_{\mathbb{P}}$ is regularly varying of index $-\alpha$ with $\alpha>1 / 2$ in the sense that there exists a regularly varying function $\tilde{f}$ (of index $-\alpha$ ) with $\tilde{f}(p)=f(p)$ for every prime $p$.

Our main result here is the following:

Theorem 2.1. Let $f \in \mathcal{M}_{c}^{2}$, such that $f \geqslant 0$ and $\left.f\right|_{\mathbb{P}}$ is regularly varying of index $-\alpha$ where $\alpha \in\left(\frac{1}{2}, 1\right)$. Then

$$
\log M_{f}(T) \sim c(\alpha) \tilde{f}(\log T \log \log T) \log T
$$


where $\tilde{f}$ is any regularly varying extension of $\left.f\right|_{\mathbb{P}}$ and

$$
c(\alpha)=\frac{B\left(\frac{1}{\alpha}, 1-\frac{1}{2 \alpha}\right)^{\alpha}}{(1-\alpha) 2^{\alpha}}
$$

and $B(x, y)=\int_{0}^{1} t^{x-1}(1-t)^{y-1} d t$ is the Beta function.

For the proof, we obtain upper and lower bounds for $\log M_{f}(T)$ which are asymptotic to each other. For the lower bounds, we require a formula for $\left\|\varphi_{f} a\right\|$ when $a \in \mathcal{M}_{c}^{2}$. This follows from the following rather elegant formula:

Lemma 2.2. For $f, g \in \mathcal{M}_{c}^{2}$,

$$
\frac{\|f * g\|}{\|f\|\|g\|}=\frac{|\langle f, g\rangle|}{\|f g\|}
$$

where $\langle\cdot, \cdot\rangle$ denotes the usual inner product for $l^{2}$.

Proof. We have

$$
\begin{aligned}
\|f * g\|^{2} & =\sum_{n=1}^{\infty}|(f * g)(n)|^{2}=\sum_{n=1}^{\infty} \sum_{c, d \mid n} f(c) \overline{f(d)} g\left(\frac{n}{c}\right) \overline{g\left(\frac{n}{d}\right)} \\
& =\sum_{c, d \geqslant 1} f(c) \overline{f(d)} \sum_{m=1}^{\infty} g\left(\frac{m[c, d]}{c}\right) \overline{g\left(\frac{m[c, d]}{d}\right)} \\
& =\sum_{m=1}^{\infty}|g(m)|^{2} \sum_{c, d \geqslant 1} f(c) \overline{f(d)} g\left(\frac{d}{(c, d)}\right) \overline{g\left(\frac{c}{(c, d)}\right)} .
\end{aligned}
$$

Collecting those terms for which $(c, d)=k$, writing $c=k m, d=k n$, and using complete multiplicativity of $f$

$$
\left(\frac{\|f * g\|}{\|g\|}\right)^{2}=\sum_{k=1}^{\infty}|f(k)|^{2} \sum_{\substack{m, n \geqslant 1 \\(m, n)=1}} f(m) \overline{f(n) g(m)} g(n) .
$$

But

$$
|\langle f, g\rangle|^{2}=\sum_{m, n \geqslant 1} f(m) \overline{f(n) g(m)} g(n)=\sum_{d=1}^{\infty}|f(d) g(d)|^{2} \sum_{\substack{m, n \geqslant 1 \\(m, n)=1}} f(m) \overline{f(n) g(m)} g(n),
$$

so the result follows.

Thus for $f, a \in \mathcal{M}_{c}^{2}$,

$$
\frac{\left\|\varphi_{f} a\right\|}{\|a\|}=\frac{\|f\| \cdot\left|\sum_{n=1}^{\infty} f(n) \overline{a_{n}}\right|}{\left(\sum_{n=1}^{\infty}\left|f(n) a_{n}\right|^{2}\right)^{1 / 2}}
$$


Since $\left|a_{n}\right| \leqslant 1$, as a corollary we have:

Corollary 2.1. For $f, a \in \mathcal{M}_{c}^{2}$,

$$
\left|\sum_{n=1}^{\infty} f(n) \overline{a_{n}}\right| \leqslant \frac{\left\|\varphi_{f} a\right\|}{\|a\|} \leqslant\|f\|\left|\sum_{n=1}^{\infty} f(n) \overline{a_{n}}\right| .
$$

Note that by complete multiplicativity,

$$
\sum_{n=1}^{\infty} f(n) \overline{a_{n}}=\prod_{p} \frac{1}{1-f(p) \overline{a_{p}}}=\prod_{p} \exp \left\{f(p) \overline{a_{p}}+O\left(\left|f(p) a_{p}\right|^{2}\right)\right\},
$$

and $\sum_{p}\left|f(p) a_{p}\right|^{2} \leqslant \sum_{p}|f(p)|^{2}=O(1)$, so that

$$
\log \frac{\left\|\varphi_{f} a\right\|}{\|a\|}=\Re \sum_{p} f(p) \overline{a_{p}}+O(1) .
$$

Proof of Theorem 2.1. We consider first upper bounds. The supremum occurs for $a \geqslant 0$ which we now assume. Write $a=\left(a_{n}\right), \varphi_{f} a=b=\left(b_{n}\right)$. Define $\alpha_{p}$ and $\beta_{p}$ for prime $p$ by

$$
\alpha_{p}=\sum_{k=1}^{\infty} a_{p^{k}}^{2} \quad \text { and } \quad \beta_{p}=\sum_{k=1}^{\infty} b_{p^{k}}^{2} .
$$

By multiplicativity of $a$ and $b$ we have $T^{2}=\|a\|^{2}=\prod_{p}\left(1+\alpha_{p}\right)$ and $\|b\|^{2}=$ $\prod_{p}\left(1+\beta_{p}\right)$. Thus

$$
\frac{\left\|\varphi_{f} a\right\|}{\|a\|}=\prod_{p} \sqrt{\frac{1+\beta_{p}}{1+\alpha_{p}}}
$$

Now for $k \geqslant 1$

$$
b_{p^{k}}=\sum_{r=0}^{k} f\left(p^{r}\right) a_{p^{k-r}}=a_{p^{k}}+f(p) b_{p^{k-1}}
$$

Thus

$$
b_{p^{k}}^{2}=a_{p^{k}}^{2}+2 f(p) a_{p^{k}} b_{p^{k-1}}+f(p)^{2} b_{p^{k-1}}^{2} .
$$

Summing from $k=1$ to $\infty$ and adding 1 to both sides gives

$$
1+\beta_{p}=1+\alpha_{p}+2 f(p) \sum_{k=1}^{\infty} a_{p^{k}} b_{p^{k-1}}+f(p)^{2}\left(1+\beta_{p}\right) .
$$

By Cauchy-Schwarz,

$$
\sum_{k=1}^{\infty} a_{p^{k}} b_{p^{k-1}} \leqslant\left(\sum_{k=1}^{\infty} a_{p^{k}}^{2} \sum_{k=1}^{\infty} b_{p^{k-1}}^{2}\right)^{1 / 2}=\sqrt{\alpha_{p}\left(1+\beta_{p}\right)}
$$


so, on rearranging

$$
\left(1+\beta_{p}\right)-\frac{2 f(p) \sqrt{\alpha_{p}\left(1+\beta_{p}\right)}}{1-f(p)^{2}} \leqslant \frac{1+\alpha_{p}}{1-f(p)^{2}} .
$$

Completing the square we find

$$
\left(\sqrt{1+\beta_{p}}-\frac{f(p) \sqrt{\alpha_{p}}}{1-f(p)^{2}}\right)^{2} \leqslant \frac{1+\alpha_{p}}{\left(1-f(p)^{2}\right)^{2}} .
$$

The term on the left inside the square is non-negative for $p$ sufficiently large since $f(p) \rightarrow 0$; in fact from $(2.4), 1+\beta_{p} \geqslant \frac{1+\alpha_{p}}{1-f(p)^{2}}$ which is greater than $\frac{f(p)^{2} \alpha_{p}}{\left(1-f(p)^{2}\right)^{2}}$ if $f(p) \leqslant 1 / \sqrt{2}$. Rearranging gives

$$
\sqrt{\frac{1+\beta_{p}}{1+\alpha_{p}}} \leqslant \frac{1}{1-f(p)^{2}}\left(1+f(p) \sqrt{\frac{\alpha_{p}}{1+\alpha_{p}}}\right) .
$$

Let $\gamma_{p}=\sqrt{\frac{\alpha_{p}}{1+\alpha_{p}}}$. Taking the product over all primes $p$ gives

$$
\frac{\left\|\varphi_{f} a\right\|}{\|a\|} \leqslant A\|f\|^{2} \prod_{p}\left(1+f(p) \gamma_{p}\right) \leqslant A^{\prime} \exp \left\{\sum_{p} f(p) \gamma_{p}\right\}
$$

for some constants $A, A^{\prime}$ depending only on $f$. (We can take $A=1$ if $f(p) \leqslant 1 / \sqrt{2}$.) Note that $0 \leqslant \gamma_{p}<1$ and $\prod_{p} \frac{1}{1-\gamma_{p}^{2}}=T^{2}$.

Let $\epsilon>0$ and put $P=\log T \log \log T$. We split up the sum on the RHS of (2.5) into $p \leqslant a P, a P<p \leqslant A P$ and $p>A P$ (for $a$ small and $A$ large). First

$$
\sum_{p \leqslant a P} f(p) \gamma_{p} \leqslant \sum_{p \leqslant a P} f(p) \sim \frac{a^{1-\alpha} P \tilde{f}(P)}{(1-\alpha) \log P}<\epsilon \tilde{f}(\log T \log \log T) \log T,
$$

for $a$ sufficiently small ${ }^{1}$. Next, using the fact that $\log T^{2}=\log \prod_{p} \frac{1}{1-\gamma_{p}^{2}} \geqslant \sum_{p} \gamma_{p}^{2}$, we have (since $\tilde{f}^{2}$ is regularly-varying of index $-2 \alpha$ )

$$
\begin{aligned}
\sum_{p>A P} f(p) \gamma_{p} & \leqslant\left(\sum_{p>A P} f(p)^{2} \sum_{p>A P} \gamma_{p}^{2}\right)^{1 / 2} \lesssim\left(\frac{2 A^{1-2 \alpha} P \tilde{f}(P)^{2} \log T}{(2 \alpha-1) \log P}\right)^{1 / 2} \\
& \sim \frac{\tilde{f}(\log T \log \log T) \log T}{A^{\alpha-1 / 2} \sqrt{\alpha-1 / 2}}<\epsilon \tilde{f}(\log T \log \log T) \log T
\end{aligned}
$$

for $A$ sufficiently large. This leaves the range $a P<p \leqslant A P$.

Note that the result follows from the case $f(n)=n^{-\alpha}$. For, by the uniform convergence theorem for regularly varying functions

$$
\left|f(p)-\left(\frac{P}{p}\right)^{\alpha} \tilde{f}(P)\right|<\epsilon f(p)
$$

\footnotetext{
${ }^{1} \mathrm{Using} \sum_{p \leqslant x} f(p) \sim \int_{2}^{x} \frac{\tilde{f}(t)}{\log t} d t \sim \frac{x \tilde{f}(x)}{(1-\alpha) \log x}$, since $\tilde{f}$ is regularly-varying of index $-\alpha$.
} 
for $a P<p \leqslant A P$ and $P$ sufficiently large, depending only on $\epsilon$. The problem therefore reduces to maximising

$$
\sum_{a P<p \leqslant A P} \frac{\gamma_{p}}{p^{\alpha}}
$$

subject to $0 \leqslant \gamma_{p}<1$ and $\prod_{p} \frac{1}{1-\gamma_{p}^{2}}=T^{2}$. The maximum clearly occurs for $\gamma_{p}$ decreasing (if $\gamma_{p^{\prime}}>\gamma_{p}$ for primes $p<p^{\prime}$, then the sum increases in value if we swap $\gamma_{p}$ and $\left.\gamma_{p^{\prime}}\right)$. Thus we may assume that $\gamma_{p}$ is decreasing.

By interpolation we may write $\gamma_{p}=g\left(\frac{p}{P}\right)$ where $g:(0, \infty) \rightarrow(0,1)$ is continuously differentiable and decreasing. Of course $g$ will depend on $P$. Let $h=\log \frac{1}{1-g^{2}}$, which is also decreasing. Note that

$$
2 \log T=\sum_{p} h\left(\frac{p}{P}\right) \geqslant \sum_{p \leqslant a P} h\left(\frac{p}{P}\right) \geqslant h(a) \pi(a P) \geqslant \operatorname{cah}(a) \log T,
$$

for $P$ sufficiently large, for some constant $c>0$. Thus $h(a) \leqslant C_{a}$ (independent of $T)$.

Now, for $F:(0, \infty) \rightarrow[0, \infty)$ decreasing,

$$
\sum_{a x<p \leqslant b x} F\left(\frac{p}{x}\right)=\frac{x}{\log x} \int_{a}^{b} F+O\left(\frac{x F(a)}{(\log x)^{2}}\right),
$$

where the implied constant is independent of $F$ (and $x$ ). For, on writing $\pi(x)=$ $\operatorname{li}(x)+e(x)$, the LHS is

$$
\begin{aligned}
\int_{a x}^{b x} F\left(\frac{t}{x}\right) d \pi(t) & =x \int_{a}^{b} \frac{F(t)}{\log x t} d t+\int_{a}^{b} F(t) d e(x t) \\
& =\frac{x}{\log \theta x} \int_{a}^{b} F+[F(t) e(x t)]_{a}^{b}-\int_{a}^{b} e(x t) d F(t) \quad(\text { some } \theta \in[a, b]) \\
& =\frac{x}{\log x} \int_{a}^{b} F+O\left(\frac{x F(a)}{(\log x)^{2}}\right),
\end{aligned}
$$

on using $e(x)=O\left(\frac{x}{(\log x)^{2}}\right)$ and the fact that $F$ is decreasing. Thus by (2.9)

$$
2 \log T \geqslant \sum_{a P<p \leqslant A P} h\left(\frac{p}{P}\right) \sim \frac{P}{\log P} \int_{a}^{A} h \sim(\log T) \int_{a}^{A} h .
$$

Since $a$ and $A$ are arbitrary, $\int_{0}^{\infty} h$ must exist and is at most 2. Also, by (2.9)

$$
\sum_{a P<p \leqslant A P} \frac{\gamma_{p}}{p^{\alpha}}=\frac{1}{P^{\alpha}} \sum_{a P<p \leqslant A P} g\left(\frac{p}{P}\right)\left(\frac{p}{P}\right)^{-\alpha} \sim \frac{P^{1-\alpha}}{\log P} \int_{a}^{A} \frac{g(u)}{u^{\alpha}} d u .
$$


Hence by (2.8),

$$
\sum_{a P<p \leqslant A P} f(p) \gamma_{p} \sim \tilde{f}(P) P^{\alpha} \sum_{a P<p \leqslant A P} \frac{\gamma_{p}}{p^{\alpha}} \sim \frac{P \tilde{f}(P)}{\log P} \int_{a}^{A} \frac{g(u)}{u^{\alpha}} d u .
$$

As $a, A$ are arbitrary, it follows from above and (2.5), (2.6), (2.7) that

$$
\log \frac{\left\|\varphi_{f} a\right\|}{\|a\|} \leqslant\left(\int_{0}^{\infty} \frac{g(u)}{u^{\alpha}} d u+o(1)\right) \tilde{f}(\log T \log \log T) \log T .
$$

Thus we need to maximize $\int_{0}^{\infty} g(u) u^{-\alpha} d u$ subject to $\int_{0}^{\infty} h \leqslant 2$ over all decreasing $g:(0, \infty) \rightarrow(0,1)$. Since $h$ is decreasing,

$$
\frac{1}{2} x h(x) \leqslant \int_{x / 2}^{x} h .
$$

The RHS can be made as small as we please for $x$ sufficiently small or large (as $\int_{0}^{\infty} h$ converges $)$. In particular, $x h(x) \rightarrow 0$ as $x \rightarrow \infty$ and as $x \rightarrow 0^{+}$. In fact, for the supremum, we can consider just those $g$ (and $h$ ) which are continuously differentiable and strictly decreasing, since we can approximate arbitrarily closely with such functions. On writing $g=s \circ h$ where $s(x)=\sqrt{1-e^{-x}}$, we have

$$
\begin{aligned}
\int_{0}^{\infty} \frac{g(u)}{u^{\alpha}} d u & =\left[\frac{g(u) u^{1-\alpha}}{1-\alpha}\right]_{0}^{\infty}-\frac{1}{1-\alpha} \int_{0}^{\infty} g^{\prime}(u) u^{1-\alpha} d u \\
& =-\frac{1}{1-\alpha} \int_{0}^{\infty} s^{\prime}(h(u)) h^{\prime}(u) u^{1-\alpha} d u=\frac{1}{1-\alpha} \int_{0}^{h\left(0^{+}\right)} s^{\prime}(x) l(x)^{1-\alpha} d x,
\end{aligned}
$$

where $l=h^{-1}$, since $\sqrt{u} g(u) \rightarrow 0$ as $u \rightarrow \infty$. The final integral is, by Hölder's inequality at most

$$
\left(\int_{0}^{h\left(0^{+}\right)} s^{1 / \alpha}\right)^{\alpha}\left(\int_{0}^{h\left(0^{+}\right)} l\right)^{1-\alpha} .
$$

But $\int_{0}^{h\left(0^{+}\right)} l=-\int_{0}^{\infty} u h^{\prime}(u) d u=\int_{0}^{\infty} h \leqslant 2$, so

$$
\int_{0}^{\infty} \frac{g(u)}{u^{\alpha}} d u \leqslant \frac{2^{1-\alpha}}{1-\alpha}\left(\int_{0}^{\infty} s^{1 / \alpha}\right)^{\alpha} .
$$

A direct calculation shows that ${ }^{2} \int_{0}^{\infty}\left(s^{\prime}\right)^{1 / \alpha}=2^{-1 / \alpha} B\left(\frac{1}{\alpha}, 1-\frac{1}{2 \alpha}\right)$. This gives the upper bound.

The proof of the upper bound leads to the optimum choice for $g$ and the lower bound. We note that we have equality in $(2.10)$ if $l /\left(s^{\prime}\right)^{1 / \alpha}$ is constant; i.e. $l(x)=c s^{\prime}(x)^{1 / \alpha}$ for some constant $c>0$ - chosen so that $\int_{0}^{\infty} l=2$. This means we take

$$
h(x)=\left(s^{\prime}\right)^{-1}\left(\left(\frac{x}{c}\right)^{\alpha}\right)=\log \left(\frac{1}{2}+\frac{1}{2} \sqrt{1+\left(\frac{c}{x}\right)^{2 \alpha}}\right) .
$$

\footnotetext{
${ }^{2}$ The integral is $2^{-1 / \alpha} \int_{0}^{\infty} e^{-x / \alpha}\left(1-e^{-x}\right)^{-1 / 2 \alpha} d x=2^{-1 / \alpha} \int_{0}^{1} t^{1 / \alpha-1}(1-t)^{-1 / 2 \alpha} d t$.
} 
from which we can calculate $g$. In fact, we show that we get the required lower bound by just considering $a_{n}$ completely multiplicative. To this end we use (2.3), and define $a_{p}$ by:

$$
a_{p}=g_{0}\left(\frac{p}{P}\right)
$$

where $P=\log T \log \log T$ and $g_{0}$ is the function

$$
g_{0}(x)=\sqrt{1-\frac{2}{1+\sqrt{1+\left(\frac{c}{x}\right)^{2 \alpha}}}},
$$

with $c=2^{1+1 / \alpha} / B\left(\frac{1}{\alpha}, 1-\frac{1}{2 \alpha}\right)$. As such, by the same methods as before, we have $\|a\|=T^{1+o(1)}$ and

$$
\log \frac{\left\|\varphi_{\alpha} a\right\|}{\|a\|}=\sum_{p} f(p) g_{0}\left(\frac{p}{P}\right)+O(1) \sim \frac{P \tilde{f}(P)}{\log P} \int_{0}^{\infty} \frac{g_{0}(u)}{u^{\alpha}} d u .
$$

By the choice of $g_{0}$, the integral on the right is $\frac{B\left(\frac{1}{\alpha}, 1-\frac{1}{2 \alpha}\right)^{\alpha}}{(1-\alpha) 2^{\alpha}}$, as required.

Remark. From the above proof, we see that the supremum (of $\left\|\varphi_{f} a\right\| /\|a\|$ ) over $\mathcal{M}_{c}^{2}$ is roughly the same size as the supremum over $\mathcal{M}^{2}$; i.e. they are logasymptotic to each other. Is it true that these respective suprema are closer still; eg. are they asymptotic to each other for $\frac{1}{2}<\alpha<1$ ?

\section{The special case $f(n)=n^{-\alpha}$}

In this case we can take $\tilde{f}(x)=x^{-\alpha}$ which is regularly varying of index $-\alpha$. Here we shall write $\varphi_{\alpha}$ for $\varphi_{f}$ and $M_{\alpha}$ for $M_{f}$.

Theorem 3.1. We have

$$
M_{1}(T)=e^{\gamma}(\log \log T+\log \log \log T+2 \log 2-1+o(1)),
$$

while for $\frac{1}{2}<\alpha<1$,

$$
\log M_{\alpha}(T)=\left(\frac{B\left(\frac{1}{\alpha}, 1-\frac{1}{2 \alpha}\right)^{\alpha}}{(1-\alpha) 2^{\alpha}}+o(1)\right) \frac{(\log T)^{1-\alpha}}{(\log \log T)^{\alpha}} .
$$

Remark. As noted in the introduction, these asymptotic formulae bear a strong resemblance to the (conjectured) maximal order of $|\zeta(\alpha+i T)|$. It is interesting to note that the bounds found here are just larger than what is known about the lower bounds for $Z_{\alpha}(T)=\max _{1 \leqslant t \leqslant T}|\zeta(\alpha+i t)|$. In a recent paper (see [8]), Lamzouri suggests $\log Z_{\alpha}(T) \sim C(\alpha)(\log T)^{1-\alpha}(\log \log T)^{-\alpha}$ with some specific function $^{3} C(\alpha)$ (for $\frac{1}{2}<\alpha<1$ ). We note that the constant appearing in (3.2) is not $C(\alpha)$ since, for $\alpha$ near $\frac{1}{2}$, the former is roughly $\frac{1}{\sqrt{\alpha-\frac{1}{2}}}$, while $C(\alpha) \sim \frac{1}{\sqrt{2 \alpha-1}}$. For $\alpha=1$, see the comment in the introduction.

\footnotetext{
${ }^{3}$ Lamzouri has the following equation $C(\alpha)=G_{1}(\alpha)^{\alpha} \alpha^{-2 \alpha}(1-\alpha)^{\alpha-1}$, where $G_{1}(x)=$ $\int_{0}^{\infty} u^{-1-1 / x} \log \left(\sum_{n=0}^{\infty} \frac{(u / 2)^{2 n}}{(n !)^{2}}\right) d u$.
} 
It would be very interesting to be able to extend these ideas (and results) to the $\alpha=\frac{1}{2}$ case. As we show in the appendix, we cannot do this by restricting $\varphi_{\frac{1}{2}}$ to smaller domains in $l^{2}$. Somehow the analogy - if such exists - between $M_{\alpha}$ and $Z_{\alpha}$ breaks down just here.

Proof of Theorem 3.1. For $\frac{1}{2}<\alpha<1$ the result follows from Theorem 2.1, so we only concern ourselves with $\alpha=1$.

For an upper bound we use $(2.5)$ with $f(p)=1 / p$ (and $A=1$ ). Thus

$$
\frac{\left\|\varphi_{1} a\right\|}{\|a\|} \leqslant \zeta(2) \prod_{p}\left(1+\frac{\gamma_{p}}{p}\right) .
$$

Again, the maximum of the RHS (subject to $0 \leqslant \gamma_{p}<1$ and $\prod_{p} \frac{1}{1-\gamma_{p}^{2}}=T^{2}$ ) occurs for $\gamma_{p}$ decreasing. Let $P=\log T \log \log T$ and $a, A$ be arbitrary constants such that $A>1>a>0$. Split the product into the ranges $p \leqslant a P, a P<p \leqslant A P$ and $p>A P$. We have

$$
\zeta(2) \prod_{p \leqslant a P}\left(1+\frac{\gamma_{p}}{p}\right) \leqslant \zeta(2) \prod_{p \leqslant a P}\left(1+\frac{1}{p}\right)=e^{\gamma}(\log a P+o(1))
$$

by Merten's Theorem, while the product over $p>A P$ is at most

$$
\exp \left\{\sum_{p>A P} \frac{\gamma_{p}}{p}\right\} \leqslant \exp \left\{\left(\sum_{p>A P} \frac{1}{p^{2}} \sum_{p>A P} \gamma_{p}^{2}\right)^{\frac{1}{2}}\right\} \leqslant \exp \left\{\sqrt{2 \log T \sum_{p>A P} \frac{1}{p^{2}}}\right\} .
$$

But $\sum_{p>A P} 1 / p^{2} \sim 1 / A P \log P \sim 1 / A \log T(\log \log T)^{2}$, so

$$
\prod_{p>A P}\left(1+\frac{\gamma_{p}}{p}\right) \leqslant 1+\frac{2}{\sqrt{A} \log \log T}
$$

for all large enough $T$. Combining the above two estimates gives

$$
\begin{aligned}
\zeta(2) & \prod_{\substack{p \\
p}}>{ }^{>A P}\left(1+\frac{\gamma_{p}}{p}\right) \leqslant e^{\gamma}\left(\log _{2} T+\log _{3} T+\log a+\frac{2}{\sqrt{A}}+o(1)\right) .
\end{aligned}
$$

For the remaining range $a P<p \leqslant A P$ we write, as before, $\gamma_{p}=g\left(\frac{p}{P}\right)$ where $g:(0, \infty) \rightarrow(0,1)$ is decreasing. Then

$$
\log \left(\prod_{a P<p \leqslant A P}\left(1+\frac{g(p / P)}{p}\right)\right) \sim \sum_{a P<p \leqslant A P} \frac{g(p / P)}{p} \sim \frac{1}{\log P} \int_{a}^{A} \frac{g(u)}{u} d u,
$$

by (2.9). Thus

$$
\frac{\left\|\varphi_{1} a\right\|}{\|a\|} \leqslant e^{\gamma}\left(\log _{2} T+\log _{3} T+\int_{a}^{A} \frac{g(u)}{u} d u-\int_{a}^{1} \frac{1}{u} d u+\frac{2}{\sqrt{A}}+o(1)\right)
$$


for all $A>1>a>0$. We need to minimise the constant term. Since $g(u)<1$, the minimum occurs for $a$ arbitrarily small. On the other hand $\int_{A}^{\infty} \frac{g(u)}{u} d u \leqslant$ $\left(\frac{1}{A} \int_{A}^{\infty} g^{2}\right)^{1 / 2}=o(1 / \sqrt{A})$, so the constant is minimized for arbitrarily large $A$; i.e. it is at most $\int_{1}^{\infty} \frac{g(u)}{u} d u-\int_{0}^{1} \frac{1-g(u)}{u} d u$. Thus

$M_{1}(T) \leqslant e^{\gamma}(\log \log T+\log \log \log T+\kappa+o(1)) \quad$ where $\kappa=\sup \{L(g): g \in G\}$.

Here $L(g)=\int_{1}^{\infty} \frac{g(u)}{u} d u-\int_{0}^{1} \frac{1-g(u)}{u} d u$ and $G$ is the set of all decreasing $g:(0, \infty) \rightarrow$ $(0,1)$ for which $\int_{0}^{\infty} \log \frac{1}{1-g^{2}} \leqslant 2$. As in the proof of Theorem 2.1, let $h=\log \frac{1}{1-g^{2}}$ so that $g=s \circ h$ where $s(x)=\sqrt{1-e^{-x}}$. Now we show $\kappa=2 \log 2-1$. Trivially, by Cauchy-Schwarz, we have

$$
L(g) \leqslant \sqrt{\int_{1}^{\infty} \frac{1}{u^{2}} d u \int_{1}^{\infty} g(u)^{2} d u} \leqslant \sqrt{\int_{0}^{\infty} h} \leqslant \sqrt{2}
$$

so $\kappa \leqslant \sqrt{2}$.

Note that the supremum is achieved for $\int_{0}^{\infty} h=2$. For if $\int_{0}^{\infty} h<2$, then we can always increase $g$ by a small amount while keeping it less than 1 and decreasing, while $\int h$ is increased by a prescribed amount - just take $g_{1}=k \circ g$ where $k:(0,1) \rightarrow(0,1)$ is increasing and $k(x)>x$. With $k(x)-x$ sufficiently small, $\int h_{1} \leqslant 2$ while $L\left(g_{1}\right)>L(g)$.

Further, we may take the supremum over $g$ for which $g$ is continuously differentiable and strictly decreasing, since they can approximate functions in $G$ arbitrarily closely.

Now, for $L(g)$ to be finite (i.e. $>-\infty)$ we need $\int_{0}^{1} \frac{1-g(u)}{u} d u$ to converge. For $x \in(0,1)$,

$$
\int_{x}^{\sqrt{x}} \frac{1-g(u)}{u} d u \geqslant(1-g(x)) \int_{x}^{\sqrt{x}} \frac{1}{u} d u=\frac{1}{2}(1-g(x)) \log \frac{1}{x} .
$$

The LHS tends to 0 as $x \rightarrow 0^{+}$, so we must have

$$
(1-g(x)) \log x \rightarrow 0 \quad \text { as } x \rightarrow 0^{+} .
$$

In particular, $g(x) \rightarrow 1$ as $x \rightarrow 0^{+}$(so $h(x) \rightarrow \infty$ as $x \rightarrow 0^{+}$). Also, as in Theorem $2.1, x h(x) \rightarrow 0$ as $x \rightarrow \infty$. Now, with $g=s \circ h$,

$$
\int_{1}^{\infty} \frac{g(u)}{u} d u=[g(u) \log u]_{1}^{\infty}-\int_{1}^{\infty} s^{\prime}(h(u)) h^{\prime}(u) \log u d u=\int_{0}^{h(1)} s^{\prime}(y) \log l(y) d y,
$$

where $l=h^{-1}$ is the inverse function of $h$. Also,

$$
\begin{aligned}
\int_{0}^{1} \frac{1-g(u)}{u} d u & =[(1-g(u)) \log u]_{0}^{1}+\int_{0}^{1} s^{\prime}(h(u)) h^{\prime}(u) \log u d u \\
& =-\int_{h(1)}^{\infty} s^{\prime}(y) \log l(y) d y .
\end{aligned}
$$

Hence $L(g)=\int_{0}^{\infty} s^{\prime} \log l$ and $\int_{0}^{\infty} l=2$. 
Now, using Jensen's inequality $\int \log f d \mu \leqslant \log \left(\int f d \mu\right)$ for $\mu$ a probability measure ([11], p.62), we have

$$
\int_{0}^{\infty} s^{\prime} \log \left(l / s^{\prime}\right)=\int_{0}^{\infty} \log \left(l / s^{\prime}\right) d s \leqslant \log \left(\int_{0}^{\infty} l / s^{\prime} d s\right)=\log \left(\int_{0}^{\infty} l\right)=\log 2 .
$$

Hence

$$
\int_{0}^{\infty} s^{\prime} \log l \leqslant \log 2+\int_{0}^{\infty} s^{\prime} \log s^{\prime}=\log 2+\int_{0}^{1} \log \left(\frac{1-u^{2}}{2 u}\right) d u=2 \log 2-1,
$$

after some calculation.

The proof of the upper bound leads to the optimum choice for $g$ and the lower bound. We note that we have equality in (3.3) if $l / s^{\prime}$ is constant; i.e. $l(x)=c s^{\prime}(x)$ for some constant $c>0-$ chosen so that $\int_{0}^{\infty} l=2$ (i.e. we take $c=2$ ). Thus, actually $\kappa=2 \log 2-1$ and the supremum is achieved for the function $g_{0}$, where

$$
g_{0}(x)=\sqrt{1-\frac{2}{1+\sqrt{1+\left(\frac{2}{x}\right)^{2}}}} .
$$

In fact, we show that we get the required lower bound by just considering $a_{n}$ completely multiplicative. To this end we use Corollary 2.1 , and define $a_{p}$ by:

$$
a_{p}=g_{0}\left(\frac{p}{P}\right)
$$

where $P=\log T \log \log T$. As such, by the same methods as before, we have $\|a\|=T^{1+o(1)}$. Let $a>0$ and $P=\log T \log \log T$. By Corollary 2.1

$$
\frac{\left\|\varphi_{1} a\right\|}{\|a\|} \geqslant \prod_{p} \frac{1}{1-\frac{a_{p}}{p}}=\prod_{p \leqslant a P} \frac{1}{1-\frac{1}{p}} \prod_{p \leqslant a P} \frac{1}{1+\frac{1-a_{p}}{p-1}} \prod_{p>a P} \frac{1}{1-\frac{a_{p}}{p}} .
$$

Using Merten's Theorem, the first product on the right is $e^{\gamma}(\log a P+o(1))$, while the second product is greater than

$$
\exp \left\{-\sum_{p \leqslant a P} \frac{1-a_{p}}{p-1}\right\} \geqslant 1-2 \sum_{p \leqslant a P} \frac{1-g_{0}(p / P)}{p} .
$$

The sum is asymptotic to $\frac{a}{\log P} \int_{0}^{a} \frac{1-g_{0}(u)}{u} d u<\frac{\epsilon}{\log P}$, for any given $\epsilon>0$, for sufficiently small $a$. The third product in (3.4) is greater than

$$
\exp \left\{\sum_{p>a P} \frac{a_{p}}{p}\right\}=\exp \left\{\frac{(1+o(1))}{\log P} \int_{a}^{\infty} \frac{g_{0}(u)}{u} d u\right\}
$$

by (2.9). Thus

$$
\frac{\left\|\varphi_{1} a\right\|}{\|a\|} \geqslant e^{\gamma}\left(\log P+\int_{a}^{\infty} \frac{g_{0}(u)}{u} d u+\log a-\epsilon\right) \geqslant e^{\gamma}\left(\log P+L\left(g_{0}\right)-\epsilon\right)
$$

for $a$ sufficiently small. As $L\left(g_{0}\right)=2 \log 2-1$ and $\epsilon$ arbitrary, this gives the required lower bound. 
Lower bounds for $\varphi_{\alpha}$ and some further speculations. We can study lower bounds of $\varphi_{\alpha}$ via the function

$$
m_{\alpha}(T)=\inf _{\substack{a \in \mathcal{M}^{2} \\\|a\|=T}} \frac{\left\|\varphi_{\alpha} a\right\|}{\|a\|}
$$

Using very similar techniques, one obtains analogous results to Theorem 3.1:

$$
\frac{1}{m_{1}(T)}=\frac{6 e^{\gamma}}{\pi^{2}}(\log \log T+\log \log \log T+2 \log 2-1+o(1))
$$

and

$$
\log \frac{1}{m_{\alpha}(T)} \sim \frac{B\left(\frac{1}{\alpha}, 1-\frac{1}{2 \alpha}\right)^{\alpha}(\log T)^{1-\alpha}}{(1-\alpha) 2^{\alpha}(\log \log T)^{\alpha}} \quad \text { for } \quad \frac{1}{2}<\alpha<1 .
$$

We see that $m_{\alpha}(T)$ corresponds closely to the conjectured minimal order of $\mid \zeta(\alpha+$ $i T) \mid$ (see [3] and [9]). We omit the proofs, but just point out that for an upper bound (for $1 / m_{\alpha}(T)$ ) we use

$$
\frac{\|a\|}{\left\|\varphi_{\alpha} a\right\|} \leqslant \prod_{p}\left(1+\frac{\gamma_{p}}{p^{\alpha}}\right)
$$

which can be obtained in much the same way as (2.5). For the lower bound, we choose $a_{p}$ as -1 times the choice in Theorem 3.1 and use Corollary 2.1.

The above formulae suggest that the supremum (respectively infimum) of $\left\|\varphi_{\alpha} a\right\| /\|a\|$ with $a \in \mathcal{M}^{2}$ and $\|a\|=T$ are close to the supremum (resp. infimum) of $\left|\zeta_{\alpha}\right|$ on $[1, T]$. One could therefore speculate further that there is a close connection between $\left\|\varphi_{\alpha} a\right\| /\|a\|$ (for such $a$ ) and $|\zeta(\alpha+i T)|$, and hence between $Z_{\alpha}(T)$ and $M_{\alpha}(T)$. Recent papers by Gonek [4] and Gonek and Keating [5] suggest this may be possible, or at least that $M_{\alpha}$ is a lower bound for $Z_{\alpha}$. On the Riemann Hypothesis, it was shown in [4] (Theorem 3.5) that $\zeta(s)$ may be approximated for $\sigma>\frac{1}{2}$ up to height $T$ by the truncated Euler product

$$
\prod_{p \leqslant P} \frac{1}{1-p^{-s}} \quad \text { for } \quad P \ll T
$$

Thus one might expect that, with $a \in \mathcal{M}_{c}^{2}+$ maximizing $\frac{\left\|\varphi_{\alpha} a\right\|}{\|a\|}$ subject to $\|a\|=T$, and $A(s)=\prod_{p \leqslant P} \frac{1}{1-a_{p} p^{-s}}($ with $P \ll T)$,

$$
\begin{aligned}
\int_{-T}^{T}|\zeta(\alpha-i t)|^{2}|A(i t)|^{2} d t & \sim \int_{-T}^{T} \prod_{p \leqslant P}\left|\left(1-\frac{p^{i t}}{p^{\alpha}}\right)\left(1-a_{p} p^{i t}\right)\right|^{-2} d t \\
& =\int_{-T}^{T} \prod_{p \leqslant P}\left|B_{p}(i t)\right|^{2} d t
\end{aligned}
$$


where $B_{p}(s)=\sum_{k \geqslant 0} b_{p^{k}} p^{-k s}$. The heuristics of Gonek and Keating now suggests this is asymptotic to

$$
2 T \prod_{p \leqslant P} \sum_{k \geqslant 0} b_{p^{k}}^{2} \sim 2 T\left\|\varphi_{\alpha} a\right\|^{2}
$$

if $P \succ \log T \log \log T$ (for the last step). Thus it would follow that

$$
Z_{\alpha}(T)^{2} \geqslant \frac{\int_{-T}^{T}|\zeta(\alpha-i t)|^{2}|A(i t)|^{2} d t}{\int_{-T}^{T}|A(i t)|^{2} d t} \sim \frac{2 T\left\|\varphi_{\alpha} a\right\|^{2}}{2 T\|a\|^{2}} \sim M_{\alpha}(T)^{2}
$$

and hence $Z_{\alpha}(T) \gtrsim M_{\alpha}(T)$.

As mentioned before, this would contradict Lamzouri's suggestion (that $\left.\log Z_{\alpha}(T) \sim C(\alpha)(\log T)^{1-\alpha}(\log \log T)^{-\alpha}\right)$ since $C(\alpha)<c(\alpha)$ (notation from Theorem 2.1) for $\alpha$ sufficiently close to $\frac{1}{2}$ at least. It is unclear to the author which possibility is more likely.

\section{Appendix}

Here we show that if $f \notin l^{2}$, we cannot hope to "capture" $\varphi_{f}$ by considering the mapping on some non-trivial subset of $l^{2}$.

Proposition A1. Suppose $\sum_{p}|f(p)|^{2}$ diverges, where $p$ ranges over the primes. Then $\varphi_{f} a \in l^{2}$ for $a \in l^{2}$ if and only if $a=0$.

Proof. Suppose there exists $a \in l^{2}$ with $a \neq 0$ such that $\varphi_{f} a \in l^{2}$. Let $a_{m}$ be the first non-zero coordinate for $a$. Let $b=\left(b_{n}\right)=\varphi_{f} a \in l^{2}$. Consider $b_{p m}$ for $p$ prime such that $p \chi_{m}$. We have

$$
b_{p m}=\sum_{d \mid p m} f(d) a_{p m / d}=a_{m} f(p)+k(p)
$$

where $k(p)=\sum_{d \mid m} f(d) a_{p m / d}$. Since

$$
\sum_{p}|k(p)|^{2} \leqslant \sum_{p}\left(\sum_{d \mid m}|f(d)|^{2} \sum_{d \mid m}\left|a_{p m / d}\right|^{2}\right) \leqslant A \sum_{d \mid m} \sum_{p}\left|a_{p m / d}\right|^{2}<\infty
$$

and $\sum_{p}\left|b_{p m}\right|^{2}$ converges, we must have

$$
\left|a_{m}\right|^{2} \sum_{p}|f(p)|^{2}<\infty
$$

This is a contradiction. 


\section{References}

[1] C. Aistleitner, K. Seip, GCD sums from Poisson integrals and systems of dilated functions, (preprint), see arXiv:1210.0741v4 [math.NT] 14 May 2013.

[2] N.H. Bingham, C.M. Goldie, J.L. Teugels, Regular Variation, Cambridge University Press, 1987.

[3] A. Granville, K. Soundararajan, Extreme values of $|\zeta(1+i t)|$, Ramanujan Math. Soc. Lect. Notes Ser 2, Ramanujan Math. Soc., Mysore (2006) 65-80.

[4] S. Gonek, Finite Euler products and the Riemann Hypothesis, Trans. Amer. Math. Soc. 364 (2012) 2157-2191.

[5] S.M. Gonek and J.P. Keating, Mean values of finite Euler products, J. London Math. Soc. 82 (2010) 763-786.

[6] T.W. Hilberdink, Determinants of Multiplicative Toeplitz matrices, Acta Arith. 125 (2006) 265-284.

[7] T.W. Hilberdink, An arithmetical mapping and applications to $\Omega$-results for the Riemann zeta function, Acta Arith. 139 (2009) 341-367.

[8] Y. Lamzouri, On the distribution of extreme values of zeta and L-functions in the strip $\frac{1}{2}<\sigma<1$, Int. Math. Res. Not. 23 (2011) 5449-5503.

[9] H.L. Montgomery, Extreme values of the Riemann zeta-function, Comment. Math. Helv. 52 (1977) 511-518.

[10] G. Pólya, Uber eine neue Weise, bestimmte Integrale in der analytischen Zahlentheorie zu gebrauchen, Göttinger Nachr. 149-159.

[11] W. Rudin, Real and Complex Analysis ( $3^{\text {rd }}$-edition), McGraw-Hill, 1986.

[12] K. Soundararajan, Extreme values of zeta and L-functions, Math. Ann. 342 (2008) 467-486.

[13] O. Toeplitz, Zur Theorie der quadratischen und bilinearen Formen von unendlichvielen Veränderlichen, Math. Ann. 70 (1911) 351-376.

[14] O. Toeplitz, Zur Theorie der Dirichletschen Reihen, Amer. J. Math. 60 (1938) 880-888.

[15] A. Wintner, Diophantine approximations and Hilbert's space, Amer. J. Math. 66 (1944) 564-578.

Address: Titus Hilberdink: Department of Mathematics, University of Reading, Whiteknights, PO Box 220, Reading RG6 6AX, UK.

E-mail: t.w.hilberdink@reading.ac.uk

Received: 30 January 2013; revised: 14 March 2013 